\title{
Caspase 11 hunts down cytosolic bacteria
}

Recently, it was shown that caspase 11 triggers pyroptosis (an inflammatory form of programmed cell death) independently of canonical inflammasome assembly. However, to date, only pathological functions for such caspase 11 activation have been reported. Now, Aachoui et al. propose that non-canonical caspase 11 activation does indeed have beneficial roles by promoting protective host immunity to cytosolic bacteria.

Aachoui et al. explored the relative contribution of various inflammasome components and of caspase 1 and caspase 11 during infection with bacteria that invade the cytoplasm. Salmonella enterica subsp. enterica serovar Typhimurium and Legionella pneumophila are taken up by macrophages but typically evade immune detection by encoding virulence factors, such as SifA and SdhA, that promote their persistence in vacuoles. However, infection with mutant

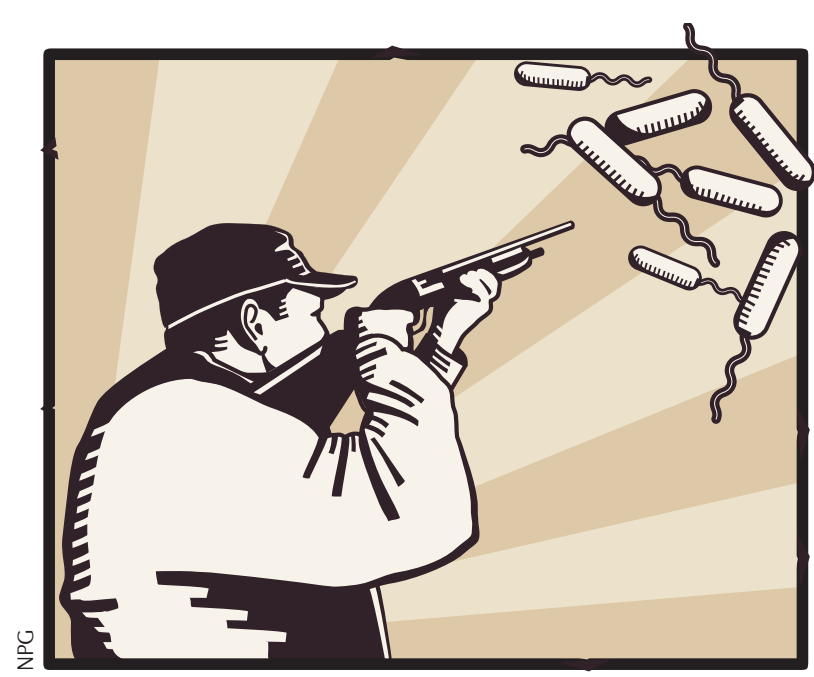

S. Typhimurium $\Delta$ sifA or L. pneumophila $\Delta s d h A$ induces rupture of vacuoles and release of the bacteria into the cytosol. Comparing infection with wild-type and mutant bacteria, the authors found that macrophages infected with $S$. Typhimurium $\Delta$ sifA showed an increase in interleukin-1 $\beta$ (IL-1 $\beta$ ) production and pyroptosis. Whereas increased IL- $1 \beta$ production was dependent on canonical inflammasome activation, the increased pyroptosis induced by S. Typhimurium $\triangle$ sifA was found to be independent of all known canonical inflammasomes. Similarly, infection with L. pneumophila $\triangle f l a A \Delta s d h A$ (which do not trigger canonical inflammasomes as they lack flagellin) induced increased levels of pyroptosis in infected macrophages in a manner independent of classical inflammasome activation.

These results suggested that the mutant bacteria were inducing pyroptosis via non-canonical activation of caspase 11. Indeed, experiments with caspase-deficient macrophages showed that caspase 11, but not caspase 1 , is required for pyroptosis following infection with $S$. Typhimurium $\Delta$ sifA or with L. pneumophila $\Delta s d h A$. The authors hypothesized that innate detection of $S$. Typhimurium $\Delta$ sifA by caspase 11 may explain why this mutant strain causes an attenuated infection in mice compared with the wild-type strain. To examine this, they co-infected wild-type or caspase 11-deficient mice with equal numbers of wild-type and mutant $S$. Typhimurium strains. Notably, 16 times as many wild-type
S. Typhimurium compared with S. Typhimurium $\Delta$ sifA bacteria were recovered from wild-type mice, but only 4 times as many wild-type compared with mutant $S$. Typhimurium bacteria were recovered from caspase 11-deficient mice. This suggests that caspase 11 promotes the clearance of $S$. Typhimurium $\triangle$ sif $A$ infection in vivo, whereas wild-type S. Typhimurium evades caspase 11 by remaining in vacuoles.

The authors also found that caspase 11 mediates innate detection and clearance of the natural cytosolic pathogens Burkholderia pseudomallei and Burkholderia thailandensis. Caspase 11-deficient mice were more acutely sensitive to infection with Burkholderia spp. and died in response to infectious doses that were not lethal to their wild-type counterparts. Aachoui et al. suggest that caspase 11 protects against lethal infection induced by bacteria that invade the cytosol and, as such, may be important for protection against ubiquitous environmental bacteria, such as $B$. thailandensis, that have not evolved to escape cytosolic detection. They further propose that this protective pyroptosis-inducing function of caspase 11 may become pathological and contribute to sepsis during overwhelming bacterial infections.

Yvonne Bordon

ORIGINAL RESEARCH PAPER Aachoui, Y. et al. Caspase-11 protects against bacteria that escape the vacuole. Science 24 Jan 2013 (doi:10.1126/ science.1230751)

FURTHER READING Bordon, Y. Innate immunity: a new path uncovers a wrongful conviction. Nature Rev. Immunol. 11, 801 (2011) 